\title{
Modeling and Trajectory Tracking Control of a Quad-rotor UAV
}

\author{
Dexin Xu, Lu Wang \\ College of Automation \\ Harbin Engineering University \\ Harbin, 150001, China \\ xudexin@hrbeu.edu.cn
}

\author{
Guangchun Li, Lidong Guo \\ College of Automation \\ Harbin Engineering University \\ Harbin, 150001, China \\ guolidong@hrbeu.edu.cn
}

\begin{abstract}
The trajectory tracking control of a quad-rotor UAV is discussed in this paper. Firstly, establish the system model by the Eula-Lagrange method. Decompound the system as the position and attitude subsystem, respectively. The backstepping approach based calculation method is proposed to obtain the expected attitude which stabilizes the position subsystem exponentially, farther more, design the controller to stabilize the attitude subsystem exponentially. The simulation results prove that this approach is able to track arbitrary trajectories of a Quad-rotor UAV.
\end{abstract}

Keywords- UAV, Trajectory tracking control, Eula-Lagrange method, Backstepping approach

\section{INTRODUCTION}

In recent years, applications and research of the unmanned aerial vehicles (UAV) has been widely concerned. Due to the characteristics of multivariate, coupled and underactuate, the control problem of the quad-rotor UAV is the emphases of the study. A lot of method has been proposed by many scholars. Rong $\mathrm{Xu}$ proposed the sliding mode control method for a class of underactuated system and applied this method into the quad-rotor UAV control[1]. David Lara proposed a method of attitude robust control[2]. Guilherme V proposed a method of robust control based on model predictive and validated the robustness of the uncertain of the model parameters[3].

However, most controllers are designed based on the predigested system model. Although this can predigest the process of the controller design, the control precision will reduce obviously. Aiming at the analysis above, a control method of nonlinear trajectory tracking is proposed based on the initial system model. This method can transfer the position control problem into the attitude control problem and predigest the control process, which stabilizes the position error by the anticipant throttle and attitude.

\section{The Principle And Model Of The System}

The quad-rotor UAV, as depicted in Figure 1, is a kind of aircrafts with the appearance of dish. It consists of four independent motor diver systems which are binded on a rigid criss-cross structure. The four rotors locate at the tips of the rigid body, and the directions of rotation of the diagonal rotors are clockwise and counterclockwise, respectively. The rates of rotation of the rotors are identical during hovering. By varying the rotor speed, one can change the lift force and create motion. Increasing or decreasing the four propellers' speeds together generates vertical motion. Changing the speeds of two propellers which are diagonal conversely produces pitch/roll motion. Yaw motion results from the difference in the counter-torque between each pair of propellers, which is caused by changing the speeds of two pair of propellers conversely.

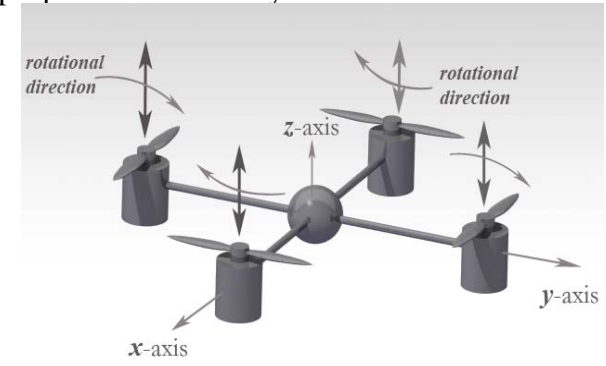

Figure 1. The principle of the quadrotor UAV

Choose the $\boldsymbol{q}^{\mathrm{T}}=\left[\begin{array}{ll}\boldsymbol{x}^{\mathrm{T}} & \boldsymbol{\eta}^{\mathrm{T}}\end{array}\right] \in \boldsymbol{R}^{6}$ as the generalized coordinate of the system state, define the Lagrange function of the system:

$$
L(\boldsymbol{q}, \dot{\boldsymbol{q}})=T(\boldsymbol{q}, \dot{\boldsymbol{q}})-V(\boldsymbol{q})
$$

Where $T(q, \dot{q})$ denotes the generalized kinetic, $V(\boldsymbol{q})$ denotes the generalized potential, $\dot{q}$ denotes the generalized velocity.

There exists the transition between $\dot{\eta}$ and $\boldsymbol{\omega}$ :

$\dot{\eta}=\phi(\eta) \omega$

$\phi(\boldsymbol{\eta})=\left[\begin{array}{ccc}1 & \sin \phi \tan \theta & \cos \phi \tan \theta \\ 0 & \cos \phi & -\sin \phi \\ 0 & \sin \phi \sec \theta & \cos \phi \sec \theta\end{array}\right]$

Define $\psi(\boldsymbol{\eta})=\phi^{-1}(\boldsymbol{\eta})$, so:

$\psi(\boldsymbol{\eta})=\left[\begin{array}{ccc}1 & 0 & -\sin \theta \\ 0 & \cos \phi & \cos \theta \sin \phi \\ 0 & -\sin \phi & \cos \theta \cos \phi\end{array}\right]$

The expression of the Lagrange function is:

$$
L(\boldsymbol{x}, \boldsymbol{\eta}, \dot{\boldsymbol{x}}, \dot{\boldsymbol{\eta}})=\frac{1}{2}\left[\begin{array}{c}
\dot{\boldsymbol{x}} \\
\dot{\boldsymbol{\eta}}
\end{array}\right]^{\mathrm{T}}\left[\begin{array}{cc}
m \boldsymbol{I}_{3} & 0 \\
0 & \psi(\boldsymbol{\eta})^{T} \boldsymbol{J} \psi(\boldsymbol{\eta})
\end{array}\right]\left[\begin{array}{c}
\dot{\boldsymbol{x}} \\
\dot{\boldsymbol{\eta}}
\end{array}\right]+m g x_{3}
$$


Where $m$ denotes the quality of the system, ${ }^{x_{3}}$ denotes the height of the UAV, $g$ denotes the acceleration of gravity $\boldsymbol{J}$ denotes the moment of inertia and assume that $\boldsymbol{J}$ is positive definite matrix.

Define the generalized input as follows:

$$
\left\{\begin{array}{l}
\boldsymbol{f}_{\xi}=\boldsymbol{R e}_{3} f-m \omega \times \dot{x} \\
\tau_{\eta}=\tau-\omega \times \boldsymbol{J} \omega
\end{array}\right.
$$

Where $\boldsymbol{f}_{\text {denotes the throttle, }} \boldsymbol{R e}_{3}$ denotes the last row of the attitude-matrix. The expression of $\boldsymbol{R}$ is:

$\boldsymbol{R}(\boldsymbol{\eta})=\left[\begin{array}{ccc}\mathrm{c} \theta \mathrm{c} \psi & -\mathrm{c} \phi \mathrm{s} \psi+\mathrm{s} \phi \mathrm{s} \theta \mathrm{c} \psi & \mathrm{s} \phi \mathrm{s} \psi+\mathrm{c} \phi \mathrm{s} \theta \mathrm{c} \psi \\ \mathrm{c} \theta \mathrm{s} \psi & \mathrm{c} \phi \mathrm{c} \psi+\mathrm{s} \phi \mathrm{s} \theta \mathrm{s} \psi & -\mathrm{s} \phi \mathrm{c} \psi+\mathrm{c} \phi \mathrm{s} \theta \mathrm{s} \psi \\ -\mathrm{s} \theta & \mathrm{s} \phi \mathrm{c} \theta & \mathrm{c} \phi \mathrm{c} \theta\end{array}\right]$

There exists the following equation based on the EulaLagrange theory:

$$
\frac{d}{d t}\left(\frac{\partial L}{\partial \dot{\boldsymbol{q}}}\right)-\frac{\partial L}{\partial \boldsymbol{q}}=\left[\begin{array}{l}
\boldsymbol{f}_{x} \\
\boldsymbol{\tau}_{\eta}
\end{array}\right]
$$

So we get the kinetics equation of the system:

$$
\left\{\begin{array}{l}
m \boldsymbol{I}_{3} \ddot{x}+m g-m \boldsymbol{\omega} \times \dot{\boldsymbol{x}}=\boldsymbol{R} \boldsymbol{e}_{3} \boldsymbol{f} \\
M(\boldsymbol{\eta}) \ddot{\boldsymbol{\eta}}+C(\boldsymbol{\eta}, \dot{\boldsymbol{\eta}}) \dot{\boldsymbol{\eta}}=\psi(\boldsymbol{\eta})^{T} \boldsymbol{\tau}
\end{array}\right.
$$

Where the expression of $M(\boldsymbol{\eta})$ and $C(\boldsymbol{\eta}, \dot{\boldsymbol{\eta}})$ is as follows[5]:

$$
\left\{\begin{array}{l}
M(\boldsymbol{\eta})=\psi(\boldsymbol{\eta})^{T} \boldsymbol{J} \psi(\boldsymbol{\eta}) \\
C(\boldsymbol{\eta}, \dot{\boldsymbol{\eta}})=\psi(\boldsymbol{\eta})^{T} \boldsymbol{J} \dot{\psi}(\boldsymbol{\eta}, \dot{\boldsymbol{\eta}})-\psi(\boldsymbol{\eta})^{T} S(\psi(\boldsymbol{\eta}) \boldsymbol{\eta}) \boldsymbol{J} \psi(\boldsymbol{\eta})
\end{array}\right.
$$

Where the arithmetic operator $S(\cdot)$ represents the asymmetry matrix of a vector.

\section{CONTROL DESIGN}

The system is a cascade system with coupled terms. Carve up the system as the position and attitude subsystems to analyze, respectively. Using the backstepping approach to design the controller. First, design the controller $f$ and virtually controller $\boldsymbol{R}$ to stabilize the position subsystem exponential and obtain the expected attitude from the $\boldsymbol{R}$ calculated above, then design the controller which stabilizes the attitude to the expected attitude exponential. The figure 2 is the control structure of the system.

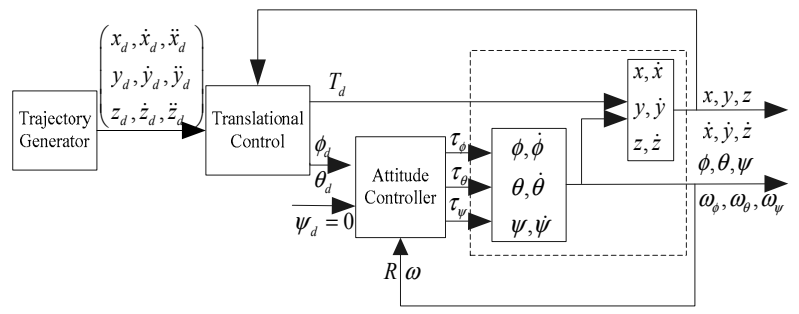

Figure 2. Control Structure of the Quad-rotor UAV

\section{A. Position subsystem control}

The purpose of the position subsystem controller is to obtain the controller $f$ and virtually controller $R$ to stabilize the position subsystem. Using the backstepping approach to design the controller to stabilize the position subsystem exponential.

Firstly, define the error between the system state and the virtually feedback:

$$
\left\{\begin{array}{l}
\boldsymbol{\varepsilon}_{1}=\boldsymbol{x}-\boldsymbol{x}_{d} \\
\boldsymbol{\varepsilon}_{2}=\dot{\boldsymbol{x}}-\dot{\boldsymbol{x}}_{d}-\alpha_{1}\left(\boldsymbol{\varepsilon}_{1}\right)
\end{array}\right.
$$

Where $\alpha_{1}$ is the virtually control volume, $\boldsymbol{x}_{d}$ and $\dot{\boldsymbol{x}}_{d}$ is the expected position and velocity, respectively, where $\dot{\boldsymbol{x}}_{d}$ is the differential of $\boldsymbol{x}_{d}$.

Define $V_{1}=\frac{1}{2}\left\|\varepsilon_{1}\right\|^{2}$, assume that $\alpha_{1}\left(\varepsilon_{1}\right)=-k_{1} \varepsilon_{1}$, then:

$\dot{V}_{1}=\boldsymbol{\varepsilon}_{1}^{\mathrm{T}} \dot{\boldsymbol{\varepsilon}}_{1}=\boldsymbol{\varepsilon}_{1}^{\mathrm{T}}\left(-\alpha_{1}\left(\boldsymbol{\varepsilon}_{1}\right)+\boldsymbol{\varepsilon}_{2}\right)=-k_{1}\left\|\boldsymbol{\varepsilon}_{1}\right\|^{2}+\boldsymbol{\varepsilon}_{1}^{\mathrm{T}} \boldsymbol{\varepsilon}_{2}$

Obviously, the $\varepsilon_{1}$ convergent to zero when $\varepsilon_{2}=0$.

$$
\begin{aligned}
& \text { Define } V_{2}=\frac{1}{2}\left\|\boldsymbol{\varepsilon}_{1}\right\|^{2}+\frac{1}{2}\left\|\boldsymbol{\varepsilon}_{2}\right\|^{2} \text {, then: } \\
& \dot{V}_{2}=-k_{1}\left\|\boldsymbol{\varepsilon}_{1}\right\|^{2}+\boldsymbol{\varepsilon}_{1}^{\mathrm{T}} \boldsymbol{\varepsilon}_{2}+\boldsymbol{\varepsilon}_{2}^{\mathrm{T}}\left(-\boldsymbol{e}_{3} m g+m \boldsymbol{\omega} \times \dot{\boldsymbol{x}}+\boldsymbol{n}_{\mathrm{d}} T_{\mathrm{d}}-\ddot{\boldsymbol{x}}_{\mathrm{d}}+k_{1}\left(\boldsymbol{\varepsilon}_{2}-k_{1} \boldsymbol{\varepsilon}_{1}\right)\right) \\
& \text { So } \quad \boldsymbol{n}_{\mathrm{d}} T_{\mathrm{d}}=\boldsymbol{e}_{3} m g-m \boldsymbol{\omega} \times \dot{\boldsymbol{x}}+\ddot{\boldsymbol{x}}_{\mathrm{d}}+\left(k_{1}^{2}-1\right) \boldsymbol{\varepsilon}_{1}-\left(k_{1}+k_{2}\right) \boldsymbol{\varepsilon}_{2}
\end{aligned}
$$

could stabilize the position subsystem. Because $\boldsymbol{n}_{\mathrm{d}}$ is the last row of the attitude matrix, we get the restriction of $\boldsymbol{n}_{\mathrm{d}}$ as $\left\|\boldsymbol{n}_{\mathrm{d}}\right\|=1$. So the expected throttle $f$ and virtually control $R$ is:

$$
\left\{\begin{array}{l}
T_{\mathrm{d}}=\left\|g \boldsymbol{e}_{3}-\boldsymbol{\omega} \times \dot{\boldsymbol{x}}+\ddot{\boldsymbol{x}}_{\mathrm{d}}-\left(k_{1}+k_{2}\right) \tilde{\boldsymbol{v}}-\left(1+k_{1} k_{2}\right) \tilde{\boldsymbol{\xi}}\right\| \\
\boldsymbol{n}_{\mathrm{d}}=\left(g \boldsymbol{e}_{3}-\boldsymbol{\omega} \times \dot{\boldsymbol{x}}+\ddot{\boldsymbol{x}}_{\mathrm{d}}-\left(k_{1}+k_{2}\right) \tilde{\boldsymbol{v}}-\left(1+k_{1} k_{2}\right) \tilde{\boldsymbol{\xi}}\right) / T_{\mathrm{d}}
\end{array}\right.
$$

Where the $k_{1}, k_{2}$ are positive constant.

Here, $\dot{V}_{2}=-k_{1}\left\|\varepsilon_{1}\right\|^{2}-k_{2}\left\|\varepsilon_{2}\right\|^{2}$, so $\boldsymbol{\varepsilon}_{1}$ and $\boldsymbol{\varepsilon}_{2}$ convergent to zero exponential.

Because $\boldsymbol{n}_{\mathrm{d}}$ has a constrain of $\left\|\boldsymbol{n}_{\mathrm{d}}\right\|=1$, so $\boldsymbol{n}_{\mathrm{d}}$ do not have the integrity information of the expected attitude. Choose $\varphi_{\mathrm{d}}=0$ to calculate the $\phi_{\mathrm{d}}$ and $\theta_{\mathrm{d}}$ :

$$
\left\{\begin{array}{l}
\phi_{\mathrm{d}}=\arcsin \left(-\boldsymbol{n}_{\mathrm{d}}(2)\right) \\
\theta_{\mathrm{d}}=\arcsin \left(\boldsymbol{n}_{\mathrm{d}}(1) / \cos \left(\phi_{\mathrm{d}}\right)\right)
\end{array}\right.
$$

So the position control problem could be converted to the attitude control problem.

\section{B. Attitude subsystem control}

The control purpose of the attitude subsystem is to obtain the control volume $\tau_{\mathrm{d}}$ to stabilize the attitude subsystem. Design the controller to stabilize the attitude subsystem exponentially by the backstepping approach. 
Firstly, define the error between the system state and the virtually feedback:

$$
\left\{\begin{array}{l}
\boldsymbol{\varepsilon}_{3}=\boldsymbol{\eta}-\boldsymbol{\eta}_{d} \\
\boldsymbol{\varepsilon}_{4}=\dot{\boldsymbol{\eta}}-\dot{\boldsymbol{\eta}}_{d}-\alpha_{2}\left(\boldsymbol{\varepsilon}_{3}\right)
\end{array}\right.
$$

Where $\alpha_{2}$ is the virtually control volume, $\boldsymbol{\eta}_{d}$ is the expected Eula attitude, $\dot{\eta}_{d}$ is the differential of $\boldsymbol{\eta}_{d}$.

$$
\begin{aligned}
& V_{3}=\frac{1}{2}\left\|\varepsilon_{3}\right\|^{2} \text {, assume that } \alpha_{2}\left(\boldsymbol{\varepsilon}_{3}\right)=-k_{3} \boldsymbol{\varepsilon}_{3} \text {, then: } \\
& \dot{V}_{3}=\boldsymbol{\varepsilon}_{3}{ }^{\mathrm{T}} \dot{\boldsymbol{\varepsilon}}_{3}=\boldsymbol{\varepsilon}_{3}{ }^{\mathrm{T}}\left(-\alpha_{2}\left(\boldsymbol{\varepsilon}_{3}\right)+\boldsymbol{\varepsilon}_{4}\right)=-k_{3}\left\|\boldsymbol{\varepsilon}_{3}\right\|^{2}+\boldsymbol{\varepsilon}_{3}{ }^{\mathrm{T}} \boldsymbol{\varepsilon}_{4}
\end{aligned}
$$

Obviously, the $\boldsymbol{\varepsilon}_{4}$ convergent to zero when $\boldsymbol{\varepsilon}_{4}=0$.

$$
\begin{aligned}
& V_{4}=\frac{1}{2}\left\|\boldsymbol{\varepsilon}_{3}\right\|^{2}+\frac{1}{2}\left\|\boldsymbol{\varepsilon}_{4}\right\|^{2}, \text { then: } \\
& \text { Define } \\
& \dot{V}_{4}=-k_{3}\left\|\boldsymbol{\varepsilon}_{3}\right\|^{2}+\boldsymbol{\varepsilon}_{3}^{\mathrm{T}} \boldsymbol{\varepsilon}_{4}+\boldsymbol{\varepsilon}_{4}^{\mathrm{T}}\left(M(\boldsymbol{\eta})^{-1}\left(\psi(\boldsymbol{\eta})^{T} \tau-C(\boldsymbol{\eta}, \dot{\boldsymbol{\eta}}) \dot{\boldsymbol{\eta}}\right)-\ddot{\boldsymbol{\eta}}_{d}\right)
\end{aligned}
$$

So the controller of the attitude subsystem is:

$$
\tau=\phi(\boldsymbol{\eta})^{T}\left(M(\boldsymbol{\eta})\left(\ddot{\boldsymbol{\eta}}_{d}-\boldsymbol{\varepsilon}_{3}-k_{4} \boldsymbol{\varepsilon}_{4}\right)+C(\boldsymbol{\eta}, \dot{\boldsymbol{\eta}}) \dot{\boldsymbol{\eta}}\right)
$$

Where $k_{3}, k_{4}$ are positive constant.

Here, $\dot{V}_{4}=-k_{3}\left\|\varepsilon_{3}\right\|^{2}-k_{4}\left\|\varepsilon_{4}\right\|^{2}$, so $\boldsymbol{\varepsilon}_{3}$ and $\boldsymbol{\varepsilon}_{4}$ convergent to zero exponential.

\section{SimUlation EXPERIMENT}

These simulations were conducted under MATLAB/Simulink, and the parameters used in simulations are: $m=0.8 \mathrm{Kg}, J_{x}=J_{y}=0.25 \mathrm{Kg} \cdot \mathrm{m}^{2}, J_{z}=0.05 \mathrm{Kg} \cdot \mathrm{m}^{2}$, $d=0.35 \mathrm{~m}$

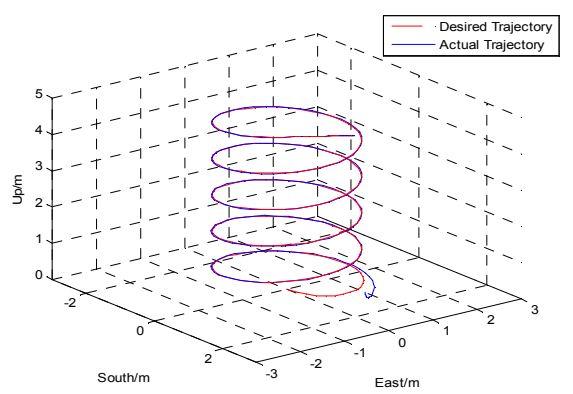

Figure 3. Trajectory Tracking of the Quad-rotor UAV

Figure 3 shows the tracking effect of an expected trajectory. Figure 4 and figure 5 show the position and attitude control effect of the quad-rotor, respectively. The proposed attitude matrix tracking controller could stabilize the attitude of the quad-rotor and finally stabilize the position error of the quad-rotor. Moreover, the control could convergent the initial position error to zero quickly.

The simulation results prove that the method proposed in this paper has the good effect of the trajectory tracking control.

\section{CONCLUSION}

The trajectory tracking control of a quad-rotor UAV is discussed is this paper. Firstly, establish the system model by the Eula-Lagrange method, and then decompound the system into two subsystems. Calculate the expected attitude from the position subsystem controller to control the attitude subsystem. The simulation results prove that the controller proposed implements the trajectory tracking control of a quad-rotor UAV.
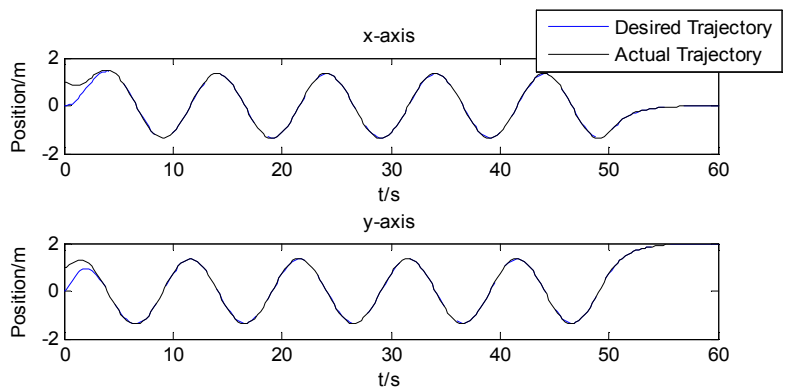

-axis

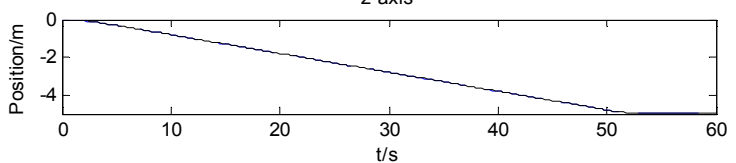

Figure 4. Position Control Effect of The Quad-rotor UAV
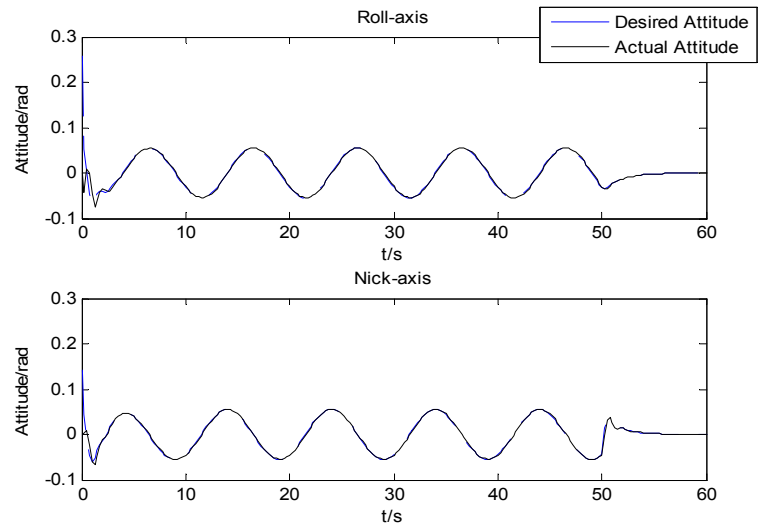

Figure 5. Attitude Control Effect of The Quad-rotor UAV

\section{ACKNOWLEDGMENT}

This work is supported by "the Fundamental Research Funds for the Central Universities"(HEUCF110404), and also supported by "International cooperation project with Russian”(2010DFR80140).

\section{REFERENCES}

[1] Rong Xu. Sliding mode control of a class of underactuated systems[J]. Automatica, 44 (2008) 233-241. 
[2] David Lara a, Gerardo Romero, Anand Sanchez. Robustness margin for attitude control of a four rotor mini-rotorcraft: Case of study[J]. Mechatronics 20 (2010) 143-152.

[3] Guilherme V. Raffo, Manuel G. Ortega, Francisco R. Rubio. An integral redictive/nonlinear control structure for a quadrotor helicopter[J]. Automatica 46 (2010) 29-39.
[4] P. Pounds, R. Mahony, P. Corke. Modelling and control of a large quadrotor robot[J]. Control Engineering Practice 18 (2010) 691-699.

[5] Reza Olfati-Saber. Nonlinear Control of Underactuated Mechanical Systems with Application to Robotics and Aerospace Vehicles [D]. Cambridge, Massachusetts: Massachusetts Institute of Technology, 2001. 